\title{
Structural Analysis Foundations for Enterprise Organizational Re-engineering
}

\author{
Azedine Boulmakoul \\ Hassan II University, FSTM \\ LIM/IST Lab. Computer Sciences Department, \\ Mohammedia Faculty of Sciences and Technology, \\ B.P. 146 Mohammedia, Morocco
}

\author{
Zineb Besri \\ Hassan II University, FSTM \\ LIM/IST Lab. Computer Sciences Department, \\ Mohammedia Faculty of Sciences and Technology, \\ B.P. 146 Mohammedia, Morocco
}

\begin{abstract}
The paper gives the investigation on organizational structure ontology. It presents a theoretical foundation for understanding organizational structure ontology by means of structural analysis. Also it discusses and provides an overview of advanced business modeling environment and enterprise modeling. The proposed solution architecture improves organizational structure of an enterprise in order to be more efficient and to be aligned with the current processes organization. The structural analysis based on Q-analysis method and using different measures like eccentricity and complexity of a system are described. It illustrates the use of structural analysis base on Q-analysis method by giving an illustration to prove how to ensure synchronization between formal organizational structure and emergent one, due to perceived changes in business processes.
\end{abstract}

\section{Keywords}

Enterprise system modeling, Q-analysis, organization structure ontology, organizational processes

\section{INTRODUCTION}

Companies, or countries, all are in a world of organizations and depend to a large extent on their adequate functioning and well-being. Companies tend to improve their structures to be more effective and efficient. To carry out change toward a structure that aligns with projects and strategies of a company, it needs to define the ontology's organization then make its structural analysis to implement a meta-model of a learning organization that aligns and meets the strategic objectives of the company. The operating mode and company's organization may vary considerably from one company to another. There are two kinds of parameters which explain this diversity, first external parameters e.g., activity and business, customers, technologies, etc. Then internal parameters e.g., history, values and culture, strategy, business processes, structures, means, methods, etc. Therefore, organizational issues are of high priority and should be of general interest. Also enterprises need to know how far their organization is intelligent.

The remaining of the paper is organized as follows: Section 2 presents organization definition. Whereas in section 3 discuss the existing enterprise modeling. Section 4 describes the proposed enterprise meta-model. Description of the proposed solution architecture in section 5. Section 6 focuses on qanalysis method for assessing the organizational structure of an enterprise. Section 7 presents an example of an organizational structure and its business process using qanalysis to improve it into a new organizational structure with less complexity. Finally, in section 8 concludes the paper and emphasizes the future works

\section{DEFINITION OF AN ORGANIZATION}

A social unit of people systematically structured and managed to meet a need or to pursue collective goals on a continuing basis. All organizations have a management structure that determines relationships between functions and positions, subdivides and delegates roles, responsibilities, and authority to carry out defined tasks. Organizations are open systems in that they affect and are affected by environment beyond their boundaries. Using the unified foundational ontology, the organization is considered as a system including organizational activities structured in business process and services, information systems supporting organizational activities [1,2] underlying information technology infrastructures and organizational structures.

Corporations are concerned about organizational structure. In other hand, a learning organization is one skilled in acquiring, creating, transferring and retaining knowledge as well as transforming that knowledge into improved performance or innovative products and services. All these activities depend on human interaction that are members on it and are, on average, intelligent and capable of learning. How can we conceive enterprise organization so as to be adapted to an intelligent and dynamic behavior? Structural analysis with its foundation and holistic practices based on algebraic topology contributes to organizational intelligence paradigm. This work permit to establish a framework for the design and development of intelligent organizations founded on advanced models of enterprise architecture and complexity management. Next some existing enterprise modeling practices.

\section{ENTERPRISE MODELING}

Enterprise models have a critical role in this study, enabling better designs for enterprises, analysis of their performances, and management of their operations. Enterprise modeling is the subject of a standards group ISO TC184 SC5 WG1 called "Industrial automation systems and integration-, Architecture, communications, and integration frameworks, Modeling and Architecture" $[3,4]$. This working group provides standards in four areas that reveal the multiplicity of necessarily views: process representation, integrating infrastructure, utilityresolving semantics, and representation of human involvement. Modeling is at once organizational, informational and human. At first study existing modeling techniques, to locate the standardization and normalization efforts $[5,6]$. 
There are many enterprise models such as, IDEF (Integrated DEFinition Methods) used for modeling activities necessary to support system analysis, design, improvement or integration [7]. Then GIM and GRAY models, here an enterprise consist of a physical system, a decision system and an information system. An enterprise can be described using four views: functional, physical, decision and informational view. Also CIMOSA (Computer Integrated Manufacturing Open Systems Architecture) defines a model-based enterprise engineering method which categorizes manufacturing operations into generic and specific functions. The advanced models are COBIT, ISO 19440 extend in the work [8], ARIS framework (Architecture of Integrated Information Systems), etc. Study has been inspired by ARIS method, it provides unified organization foundation ontology.

ARIS is an approach to enterprise modeling. It offers methods for analyzing processes and taking a holistic view of process design, management, workflow, and application processing. See works given in [9]. ARIS enterprise architecture framework defines organization as a system including: organizational activities structured in business processes and services; Information system supporting organizational activities; Information technology infrastructures and Organizational structures. Organizational view in the requirement definition layers includes modeling concepts for the enterprise's structure.

\section{PROPOSED META-MODEL}

Inspired from ISO19440 extended and ARIS to conceive the enterprise meta-model. Figure1 shows fragments of the proposed organizational meta-model. It defines the following packages. Organizational package (green classes) which includes the generic classes: Organizational Unit: entity responsible for achieving organizational goals; Position: the smallest organizational unit; Performer: represents a person assigned to an organization; Location: a geographical location of an organization unit, person, position or organization cell; Then Objective package (cyan classes) that introduces Objective: include explicit goals and targets set by the enterprise, while indicators are associated with assessing the enterprise's progress towards its objective. Finally Process and Resource packages (rest of class) which define Activity: the fundamental business entities that represents actions taken by the enterprise. Activities can be composed of sub-activities thus can be combined with other business to represent business Process; besides Resources: business entities that can be used or consumed during the performance of an activity.

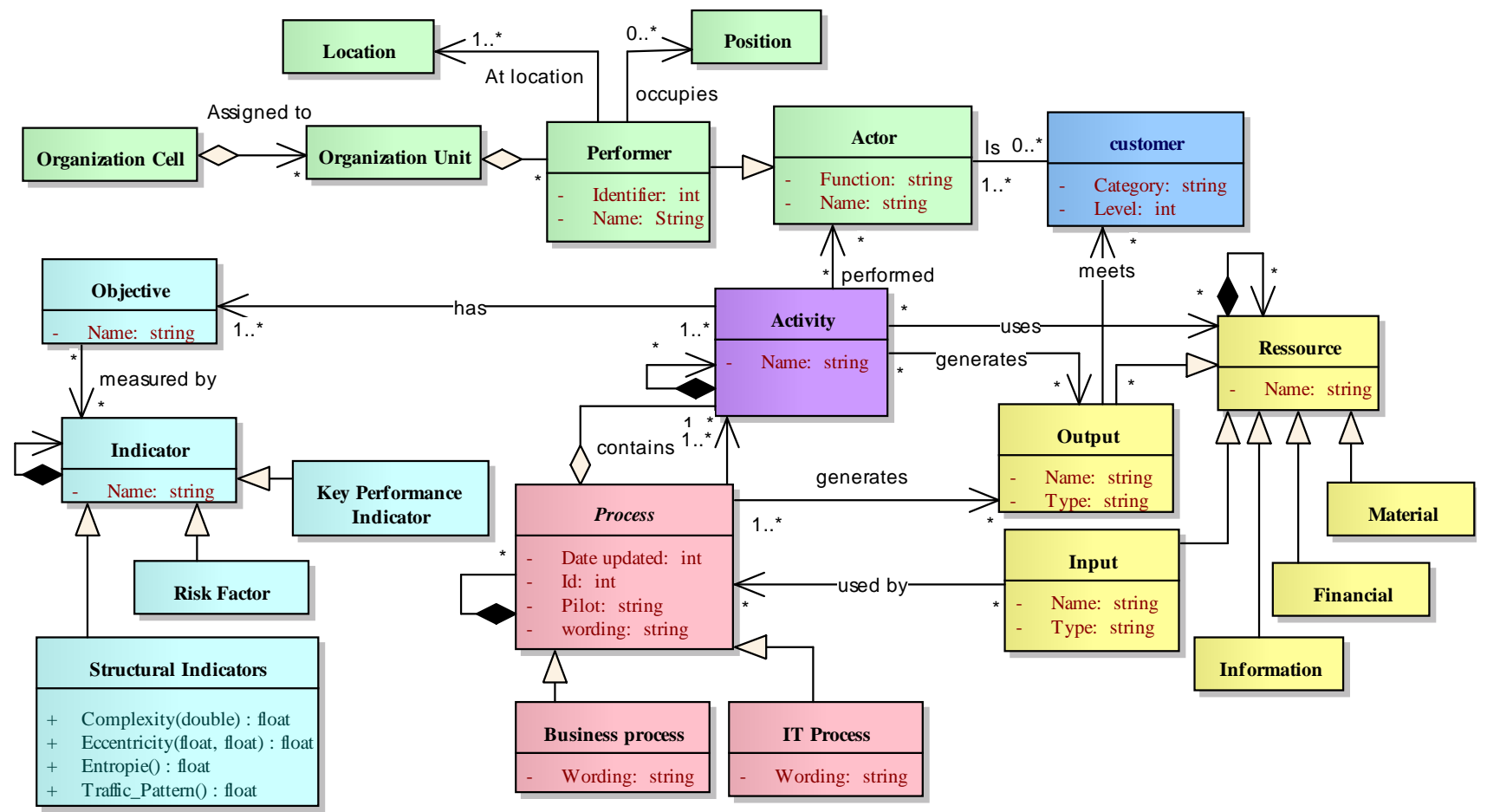

Fig 1: Proposed Meta-Model for Enterprise Architecture 


\section{SOLUTION ARCHITECTURE}

The proposed system architecture for an intelligent organization based on the structural analysis framework is organized in five layers: Repository, Extractor, Structural Analysis and Viewer and Organizational Structure Database. In the following it describes each layer of the proposed architecture shown in figure 2 .

-Repository includes organization structure, processes, activities and different kind of resources. Using best practices in business and enterprise management like COBIT, CIMOSA, etc. This layer will be the input of the proposed system.

-SETL, Structural Extract Transform and Load, allows us to extract cleaned and useful information for a given analysis. It also provides the possibility to visualize the result of SETL processing.

-Structural Analysis Framework is the aim layer in the proposed system architecture. It takes as input the extract useful information from the repository so as to do structural analysis. It consists of a measure of complexity, eccentricity and other organizational indicators in order to make a diagnosis of the current state of the enterprise organization and see if it is stable or requires improvement to make it more stable and aligned with the enterprise goal.

- Viewer/Selector to display and show the results of the structural analysis framework.

- Organizational structure database where saving data and future results of new stable organizational structures

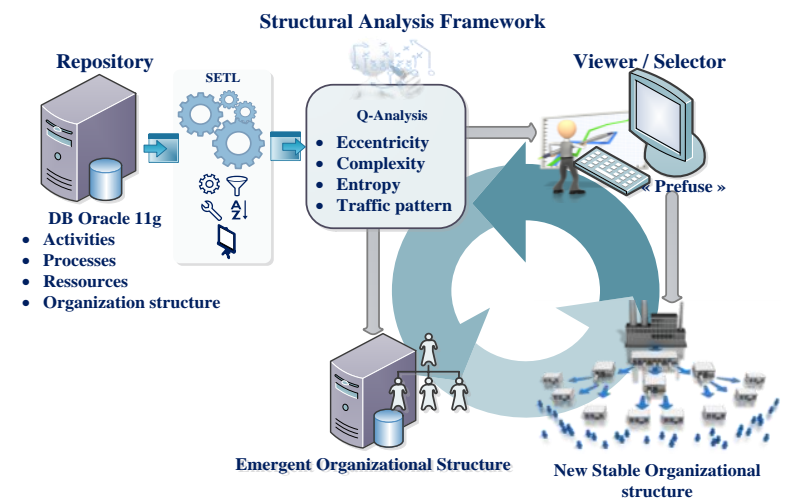

Fig 2: Solution Architecture

\section{STRUCTURAL ANALYSIS OF A BUSINESS PROCESS}

The concept of structural analysis of a business enterprise is a simple notion of showing the user different views of an enterprise: who does what, where and how. It provides an interactive, analytical environment for a user to view the different entities in an enterprise in many ways [13]. It focuses on Q-analysis method to improve the organizational structure of an enterprise.

\subsection{Preliminaries}

Let $\mathrm{P}$ be a set of processes and $\mathrm{R}$ is a set of resources. $\mathrm{D}$ is a database of business processes (BP), where each BP has a unique identifier (rid) and contains a set of processes. The set of all rids is denoted as $\mathrm{R}$. The input database is a binary relation $\lambda \subseteq$ PxR. The example given in Table I represents an illustration of the database and its adjacency matrix of a BP.
Table 1. Structural presentation of business process

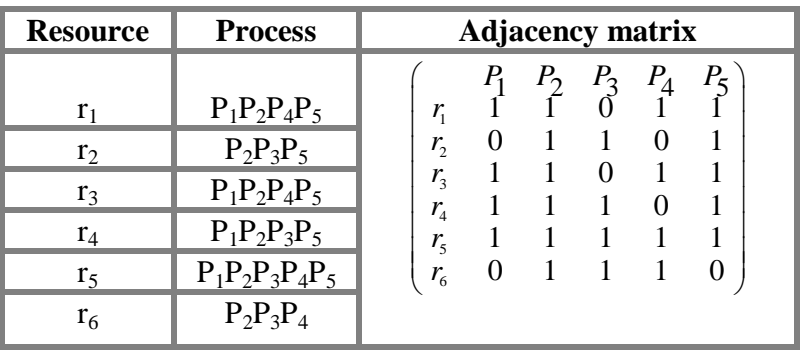

\subsection{Algebraic-topology framework}

Algebraic topology is a branch of mathematics in which tools from abstract algebra are used to study topological spaces [1113]. The basic method now applied in algebraic topology is to investigate spaces via algebraic invariants. For example, by mapping them, two groups which have a great deal of manageable structure in a way that respects the relation of homeomorphism of spaces. This allows one to recast statements about topological spaces into statements about groups, which are often easier to prove. Algebraic topology adds algebraic tools [13].

\subsection{Simplicial complexes}

A finite simplicial complex $\mathrm{K}$ is a set of vertices, $X=\left\{x_{1} \ldots x_{n}\right\}$ and a set of subsets of $X$. The subset $\sigma p i$ with $\mathrm{p}+1$ vertices is called a p-simplex. $\sigma_{\mathrm{pi}}$ is said to have dimension $\mathrm{p}$ (one less than the number of vertices). The superscript $\mathrm{i}$ is an index (more than one simplex has dimension p). A simplex $\sigma_{\mathrm{q}}$ is said to be a q-dimensional face of $\sigma_{p}$, if and only if every vertex of $\sigma_{q}$ is also a vertex of $\sigma_{p}$. K satisfies the condition that all the faces of its simplicies are also in $\mathrm{K}$. The dimension of $\mathrm{K}$ is the largest value of $\mathrm{p}$ for which there exists $\sigma_{\mathrm{pi}}$. The simplicies can be represented by a spatial structure usually shown as a polyhedral one. Gluing such polyhedra of mixed dimension forms the complex [1416].

A complex $\mathrm{KY}(\mathrm{X} ; \lambda)$ can be represented in Euclidean space $\mathrm{E}^{\mathrm{H}}$ in the following way, for a suitable choice of $\mathrm{H}$. Each $\mathrm{p}$ simplex, typically $\sigma_{\mathrm{p}}=\left\langle\mathrm{x}_{1}, \ldots, \mathrm{x}_{\mathrm{p}+1}\right\rangle$, is made to correspond to a convex polyhedron in $\mathrm{E}^{\mathrm{H}}$ with $(\mathrm{p}+1)$ vertices which themselves correspond to $\mathrm{x}_{1}, \ldots, \mathrm{x}_{\mathrm{p}+1}$. Thus, in an intuitive sense, in $E^{\mathrm{H}}$ the simplex $\sigma_{\mathrm{p}}$ is represented by the solid polyhedron with $(p+1)$ vertices. The complex $K$ is then represented by collection of polyhedra suitably connected to each other by sharing faces (or sub-polyhedra).

\subsection{Chains of q-connection in $\mathbf{K}$}

Given two simplicies $\sigma_{\mathrm{p}}, \sigma_{\mathrm{r}}$ in $\mathrm{K}$ they are joined by a chain of connection if there exists a finite sequence of simplicies $\sigma_{\alpha 1}, \sigma_{\alpha 2}, \ldots, \sigma_{\alpha \mathrm{h}}$ such that : (i): $\sigma_{\alpha 1} \leq \sigma_{\mathrm{p}}$; (ii): $\sigma_{\alpha \mathrm{h}} \leq \sigma_{\mathrm{r}}$; (iii): $\sigma_{\alpha \mathrm{i}}$, $\sigma_{\alpha i+1}$ share a common face (say) $\sigma_{\beta i}(i=1, \ldots, h-1)$.This sequence is a chain of q-connection (q-connectivity) if $\mathrm{q}$ is the least of the integers $\sigma_{\alpha 1}, \sigma_{\alpha 2}, \ldots, \sigma_{\alpha h}$. The length of the chain will be taken as (h-1) and when needed the chain may be denoted by $\left[\sigma_{\mathrm{p}}, \sigma_{\mathrm{r}}\right]_{\mathrm{q}}$

\subsection{Q-analysis}

The Q-analysis is based on the q-nearness and q-connectivity relations between the simplicies of a given complex (or simplicial complex) $[3,17,18]$. A Q-analysis of a complex K determines the number of distinct equivalence classes, or qconnected components, for each level of dimension q ranging from 0 to $\mathrm{q}-1$. The equivalence classes are decided by a rule 
as follows. If two simplicies are q-connected (either q-near or $\mathrm{q}$-connected), then they are in a same class. To see this it introduces, for a fixed $\mathrm{q}$, a relation $\gamma_{\mathrm{q}}$ on the simplicies of $\mathrm{K}$, defined by: $\left(\sigma_{\mathrm{p}}, \sigma_{\mathrm{r}}\right) \in \gamma_{\mathrm{q}}$ if and only if $\sigma \mathrm{p}$ is q-connected to $\sigma_{\mathrm{r}}$. This $\gamma_{q}$ is reflexive, symmetric and transitive and therefore an equivalence relation. The equivalence classes, under $\gamma_{q}$ are the members of the quotient set $\mathrm{K} / \gamma_{\mathrm{q}}$, and constitutes a partition of all simplicies of $\mathrm{K}$ which are of order $\geq \mathrm{q}$. Denote the cardinality of $\mathrm{K} / \gamma \mathrm{q}$ by Qq. This equals the number of distinct $\mathrm{q}$-connected components in $\mathrm{K}$. When we analyze $\mathrm{K}$ by finding all the values of $\mathrm{Q}_{0}, \mathrm{Q}_{1}, \mathrm{Q}_{2}, \ldots, \mathrm{Q}_{\mathrm{N}}$ where $\mathrm{N}=\operatorname{dimK}$, we say that we have performed a $\mathrm{Q}$-analysis on $\mathrm{K}$. To find the shared face q-value between all pairs of the Y's in $\mathrm{KY}(\mathrm{X} ; \lambda)$., the following steps could be performed: (i) form $\Lambda \mathrm{x} \Lambda \mathrm{T}$, (ii) evaluate $\Lambda \times \Lambda \mathrm{T}-\Omega=\left(\omega_{\mathrm{ij}}\right)$ and $\omega_{\mathrm{ij}}=1$. For example, the Q-analysis of the complex in example 1 leads to the following equivalence classes at the different dimensional levels of $\mathrm{q}=0$, $\mathrm{q}=1, \mathrm{q}=2$ and $\mathrm{q}=3$. Each equivalence class is enclosed in the curly brackets. The sign "-" in the matrix stands for -1 , and shows that $\mathrm{y}_{1}$ and $\mathrm{y}_{4}$ are disconnected.

Table 2. Q-Analysis of $\mathrm{KY}(\mathrm{X}, \lambda)$ given in example 1

\begin{tabular}{|c|c|c|c|c|c|c|c|}
\hline $\begin{array}{r}\text { At } q=3 \text { it has } Q_{3}=1 ; \\
\left\{y_{1}\right\} \\
\text { At } q=2 \text { it has } Q_{2}=4 ; \\
\left\{y_{1}\right\},\left\{y_{2}\right\},\left\{y_{4}\right\},\left\{y_{6}\right\} \\
\text { At } q=1 \text { it has } Q_{1}=4 ; \\
\left\{y_{1}, y_{2}\right\},\left\{y_{3}\right\},\left\{y_{4}, y_{6}\right\} \\
\left\{y_{5}\right\} \\
\text { At } q=0 \text { it has } Q_{0}=1 ; \\
\text { all }\left\{y_{1}, y_{2}, y_{3}, y_{4}, y_{5}, y_{6}\right\}\end{array}$ & $\Lambda \times \Lambda^{\mathrm{T}}-\Omega=$ & $\begin{array}{ccc}\mathrm{y}_{1} & \mathrm{y}_{2} & \mathrm{y} \\
3 & 1 & \\
& 2 & \end{array}$ & $\begin{array}{l}\mathrm{y}_{3} \\
- \\
0 \\
1\end{array}$ & $\begin{array}{c}y_{4} \\
- \\
- \\
0 \\
2\end{array}$ & $\begin{array}{l}\mathrm{y}_{5} \\
0 \\
0 \\
- \\
0 \\
1\end{array}$ & $\begin{array}{l}y_{6} \\
0 \\
0 \\
0 \\
1\end{array}$ & $\begin{array}{l}\mathrm{y}_{1} \\
\mathrm{y}_{2} \\
\mathrm{y}_{3} \\
\mathrm{y}_{4} \\
\mathrm{y}_{5} \\
\mathrm{y}_{6}\end{array}$ \\
\hline
\end{tabular}

7. STRUCTURAL ANALYSIS EXAMPLE

It proposes an example of business process to capitalize on Qanalysis method for organization structure assessment. It has four organization units with six performers. Using ARIS ontology and ARIS platform for drawing the organizational structure shown in figure 3 . The business process's example supports five activities using all performers (figure 4).

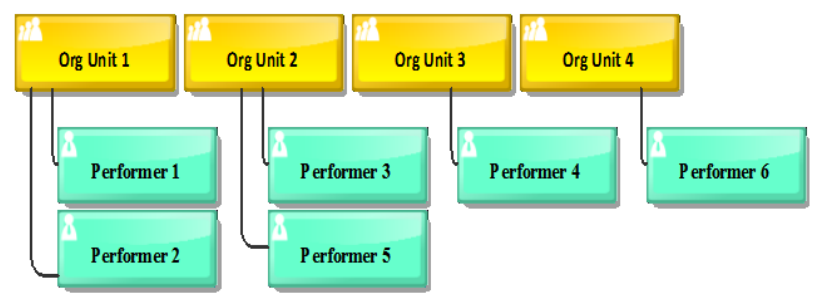

Fig 3: Organizational structure example

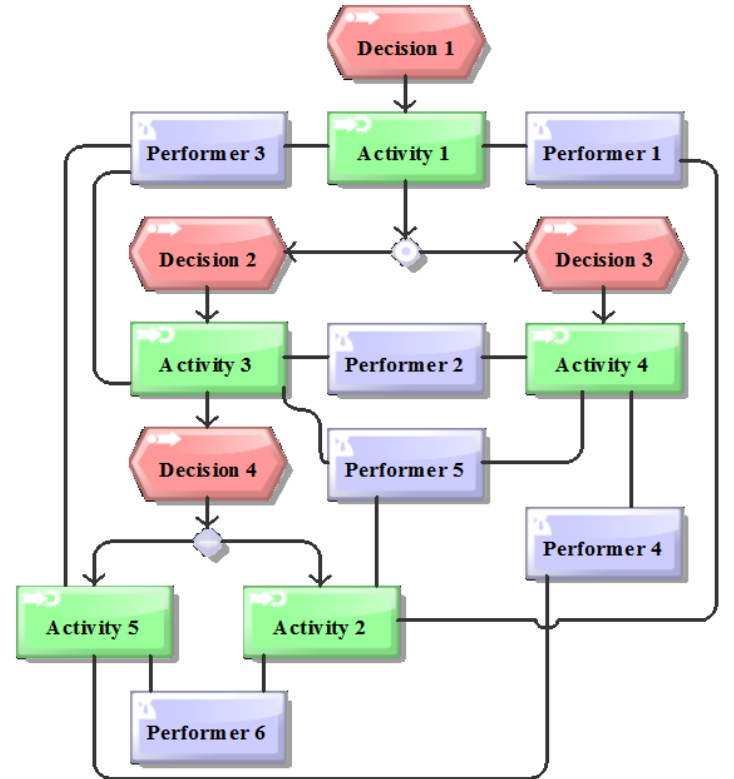

Fig 4: Example of business process

Using q-analysis method for extracting the adjacency matrix $\Lambda=(\mathrm{P} \times \mathrm{A})$. The Q-analysis of the complex in example (Table 3 ) leads to the following equivalence classes at the different dimensional levels of $\mathrm{q}=0, \mathrm{q}=1$ and $\mathrm{q}=2$. Each equivalence class is enclosed in the curly brackets (Tables 4 and 6).

Table 3. Adjacency matrix of the proposed business process

\begin{tabular}{|c|c|c|c|c|c|c|c|}
\hline Performers & Activities & \multicolumn{6}{|c|}{ Adjacency matrix } \\
\hline $\mathrm{P}_{1}$ & $\mathrm{~A}_{1} \mathrm{~A}_{2}$ & \multirow{6}{*}{\multicolumn{2}{|c|}{$\begin{array}{ll} & \mathrm{A}_{1} \\
\mathrm{P}_{1} & 1 \\
\mathrm{P}_{2} & 0 \\
\mathrm{P}_{3} & 1 \\
\mathrm{P}_{4} & 0 \\
\mathrm{P}_{5} & 0 \\
\mathrm{P}_{6} & 0\end{array}$}} & \multirow{6}{*}{$\begin{array}{c}\mathrm{A}_{2} \\
1 \\
0 \\
0 \\
0 \\
1 \\
1\end{array}$} & \multirow{6}{*}{$\begin{array}{c}\mathrm{A}_{3} \\
0 \\
1 \\
1 \\
0 \\
1 \\
0\end{array}$} & \multirow{6}{*}{$\begin{array}{c}\mathrm{A}_{4} \\
0 \\
1 \\
0 \\
1 \\
1 \\
0\end{array}$} & $\begin{array}{c}\mathrm{A}_{5} \\
0\end{array}$ \\
\hline $\mathrm{P}_{2}$ & $\mathrm{~A}_{3} \mathrm{~A}_{4}$ & & & & & & 0 \\
\hline $\mathrm{P}_{3}$ & $\mathrm{~A}_{1} \mathrm{~A}_{3} \mathrm{~A}_{5}$ & & & & & & 1 \\
\hline $\mathrm{P}_{4}$ & $\mathrm{~A}_{4} \mathrm{~A}_{5}$ & & & & & & 1 \\
\hline $\mathrm{P}_{5}$ & $\mathrm{~A}_{2} \mathrm{~A}_{3} \mathrm{~A}_{4}$ & & & & & & 1 \\
\hline $\mathrm{P}_{6}$ & $\mathrm{~A}_{2} \mathrm{~A}_{5}$ & & & & & & \\
\hline
\end{tabular}

Table 4. Q-Analysis of business process sample

\begin{tabular}{|c|c|c|}
\hline $\begin{array}{l}\text { At } \mathrm{q}=2 \text { it has } \mathrm{Q}_{2}=2 ;\left\{\mathrm{P}_{3}\right\}\left\{\mathrm{P}_{5}\right\} \\
\text { At } \mathrm{q}=1 \text { it has } \mathrm{Q}_{1}=5 ;\left\{\mathrm{P}_{1}\right\}\left\{\mathrm{P}_{2} \text {, }\right. \\
\left.\mathrm{P}_{5}\right\}\left\{\mathrm{P}_{4}\right\}\left\{\mathrm{P}_{6}\right\}\left\{\mathrm{P}_{3}\right\} \\
\text { At } \mathrm{q}=0 \text { it has } \mathrm{Q}_{0}=1 ; \text { all } ;\left\{\mathrm{P}_{1} \text {, }\right. \\
\left.\mathrm{P}_{2}, \mathrm{P}_{3}, \mathrm{P}_{4}, \mathrm{P}_{5}, \mathrm{P}_{6}\right\} \\
\qquad \begin{array}{c}210 \\
\qquad=\{251\}\end{array}\end{array}$ & $\Lambda \times \Lambda^{\mathrm{T}}-\Omega=$ & 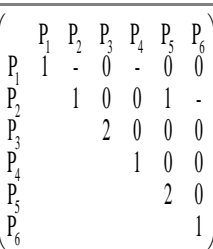 \\
\hline
\end{tabular}

It founds 3 equivalences classes $\mathrm{Q}_{2}, \mathrm{Q}_{1}$ and $\mathrm{Q}_{0}$ for each class with a number of simplex embedded (Table 4). 


\subsection{Eccentricity:}

It describes the status of an individual simplex within the entire complex. It indicates the degree of integration of a specific simplex into the whole complex K. [14] suggests a measure of eccentricity; [12] defines the conventional measure of eccentricity for a simplex and denoted as ecc (equation 1):

$$
\operatorname{ecc}(\sigma)=\frac{\hat{q}-\breve{q}}{\check{q}+1}
$$

Where top-q " $\hat{q}$ " is the dimensional level at which a simplex first appears in the simplicial complex. Bottom-q "q" "is the level at which simplex first becomes connected in a component with another simplex. A simplex is eccentric when it is badly embedded within the complex. $[17,19,22]$ suggest another measure of eccentricity called ecc' (equation 2 )

$$
\operatorname{ecc}^{\prime}(\sigma)=\frac{2 \sum_{i} q_{i} / \sigma_{i}}{q_{\max }\left(q_{\max }+1\right)}
$$

Where $q_{i}$ each q-level where $\sigma$ appears, $\sigma i$ is the number of elements in $\sigma_{i}$ 's equivalence class at level $q_{i}$ and $q_{\max }$ the maximum level of the complex. In the proposed business process example and using eccentricity measures it founds the following results.

The difference between ecc and ecc' is that ecc depends on the other simplicies and takes values in the interval of $[0, \infty]$.

\subsection{Performer eccentricity}

It observes that the simplex $\sigma_{1}\left(\mathrm{P}_{2}\right)$ has an eccentricity $\operatorname{ecc}\left(\sigma_{1}\left(P_{2}\right)\right)=0$ i.e. the performer $P_{2}$ is very connected to its activity and Organizational unit. Also for $\mathrm{P}_{5}$ it has an eccentricity with a value of 0,5 which mean the performer $P_{5}$ is relatively connected to its activities and organizational units. However, the performer $\mathrm{P}_{3}$ is the most eccentric one because it has the high value of the measure $\operatorname{ecc}\left(\sigma_{2}\left(\mathrm{P}_{3}\right)\right)=2$. It concludes that the performer $\mathrm{P}_{3}$ is dispatched between all activities and organizational unit. The second measure ecc' shows the eccentricity between simplexes. It also confirms that the performer $\mathrm{P}_{3}$ is the eccentric one among the other performers.

Table 5. Eccentricity of each performer

\begin{tabular}{|c|c|c|}
\hline Simplex & Ecc & ecc' \\
\hline$\sigma_{1}\left(\mathrm{P}_{1}\right)$ & 1 & 1 \\
\hline$\sigma_{1}\left(\mathrm{P}_{2}\right)$ & 0 & 0.5 \\
\hline$\sigma_{2}\left(\mathrm{P}_{3}\right)$ & 2 & 1.33 \\
\hline$\sigma_{1}\left(\mathrm{P}_{4}\right)$ & 1 & 1 \\
\hline$\sigma_{2}\left(\mathrm{P}_{5}\right)$ & 0.5 & 0.83 \\
\hline$\sigma_{1}\left(\mathrm{P}_{6}\right)$ & 1 & 1 \\
\hline
\end{tabular}

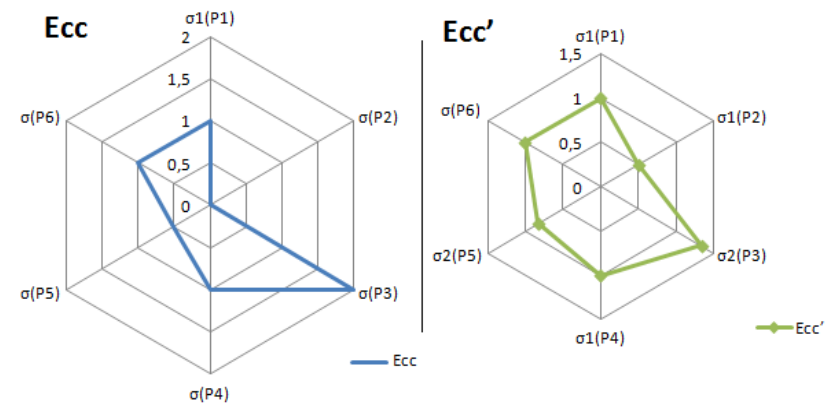

Fig 5: Eccentricities (ecc and ecc') of each performer

\subsection{Activity eccentricity}

Observe that the simplex $\sigma_{1}\left(\mathrm{~A}_{3}\right)$ and $\sigma_{1}\left(\mathrm{~A}_{4}\right)$ have the same value of eccentricity $\operatorname{ecc}\left(\sigma_{1}\left(\mathrm{~A}_{3}\right)\right)=\operatorname{ecc}\left(\sigma_{1}\left(\mathrm{~A}_{3}\right)\right)=0.5$ i.e. activities $\mathrm{A}_{3}$ and $\mathrm{A}_{4}$ are very connected to their Performers and Organizational unit. For $A_{1}$ it has an eccentricity with a value of 1 which means that activity $A_{1}$ is relatively connected to its performers and organizational units. However, activities $\mathrm{A}_{2}$ and $\mathrm{A}_{5}$ are the most eccentric one because they have the high value of the measure ecc $\left(\sigma_{2}\left(\mathrm{~A}_{2}\right)\right)=\operatorname{ecc}\left(\sigma_{2}\left(\mathrm{~A}_{2}\right)\right)=2$.

Table 6. Q-Analysis of activities

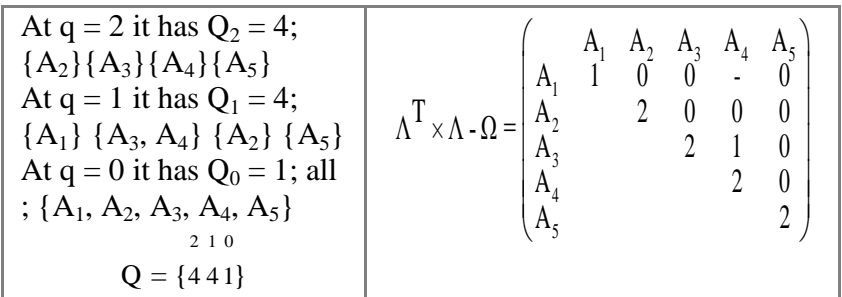

Table 7. Eccentricity of each activity

\begin{tabular}{|c|c|c|}
\hline Simplex & ecc & Ecc \\
\hline$\sigma_{1}\left(\mathrm{~A}_{1}\right)$ & 1 & 1 \\
\hline$\sigma_{2}\left(\mathrm{~A}_{2}\right)$ & 2 & 1 \\
\hline$\sigma_{2}\left(\mathrm{~A}_{3}\right)$ & 0.5 & 0.416 \\
\hline$\sigma_{2}\left(\mathrm{~A}_{4}\right)$ & 0.5 & 0.416 \\
\hline$\sigma_{2}\left(\mathrm{~A}_{5}\right)$ & 2 & 1 \\
\hline
\end{tabular}
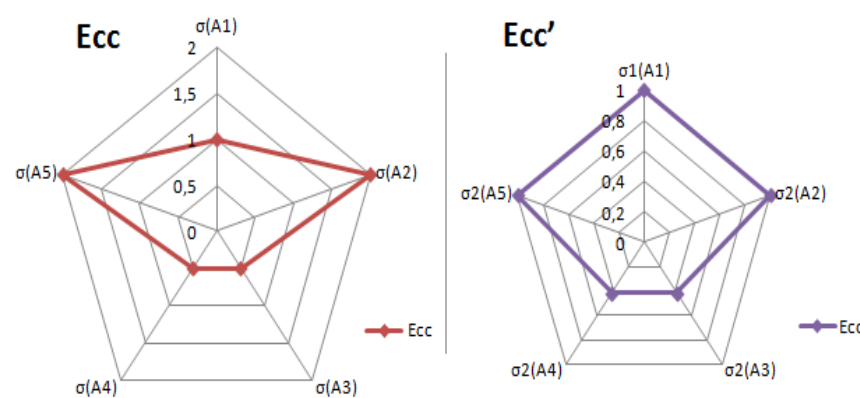

Fig 6: Eccentricities (ecc and ecc') of each activity

\subsection{Complexity}

The complexity of the system structure of this example can be described by the complexity measure $\Psi$ (K) suggested by [23] in the equation 3 :

$$
\Psi(K)=2\left[\sum_{\mathrm{k}=0}^{\text {dimk }}(\mathrm{k}+1) \mathrm{Q}_{\mathrm{k}} /(\operatorname{dimK}+1)(\operatorname{dim} \mathrm{K}+2)\right]
$$

Where $\mathrm{Q}_{\mathrm{k}}$ is the $\mathrm{k}^{\text {th }}$ component of $\mathrm{Q}$. In the proposed example the complexity of a system's structure is:

Table 8. Complexity measure for performers and activities

\begin{tabular}{|c|c|}
\hline Activity & Complexity \\
210 & $\Psi(\mathrm{K})=0.93$ \\
$\mathrm{Q}=\{441\}$ & $\Psi(\mathrm{K})=2.26$ \\
\hline Performers & 210 \\
$\mathrm{Q}=\{251\}$ & \\
\hline
\end{tabular}

See that organizational unit 2 does all activities in the business process. It consolidates performer $\mathrm{P}_{6}$ in Org-unit4 with Orgunit2 who shares activities in Org-unit2. After re-engineering of organizational structure, It has a new Adjacency matrix and new values of Q-analysis as follows. 
[13] Hocking, J. G. and Young G. S, (1988). Topology. New York, Dover Publications.

[14] Atkin, R, (1974). Mathematical Structure in Human Affairs. London, Heinemann.

[15] Atkin, R, (1977). Combinatorial Connectivities in Social Systems. Basel, Birkhäuser Verlag.

[16] Tsau Lin , Y, I.J. Chiang. (2005). A simplicial complex, a hyper graph, structure in the latent semantic space of document clustering. International journal of approximate Reasoning 40.55-80.

[17] Duckstein, L, P. Bartels, and H. Weber, J. E, (1988). Organization of a knowledge base by Q-analysis. Applied Mathematics and Computation vol. 26(4), 289301.

[18] Jiang, B, I. Omer, (2006). Spatial Topology and its Structural Analysis based on the concept of Simplicial Complex. 9th AGILE Conference on Geographic Information Science, Visegrad, Hungary 204-212.

[19] Beaumont,J.R, A.C. Gatrell, An introduction To QAnalysis. ISSN 0306-6142.
[20] Masakul, K, S. Thanawastien, P. Sermsuk, (2007). Ontological Automation of Strategic information System Planing. 24th South east Asia Regional computer conference, November. Bangkok, Thailand.

[21] Linton,C, Freeman, (1980). Q-Analysis and the structure of friendship networks. International journal ManMachine studies 12, 367-378.

[22] Duckstein, L, A. Steven, Nobe, (1997). Q-analysis for modeling and decision making. European journal of operational Research 103 411-425.

[23] Jiang, B. and Omer I, (2006). Spatial Topology and its Structural Analysis based on the Concept of Simplicial Complex, 9th AGILE Conference on Geographic Information Science, Visegrád, Hungary, pp. 204-212.

[24] Chin, C, L. Duckstein, M.L. Wymore, (1991). Factory automation project selection using multi-criterion Qanalysis. Applied Mathematics and Computation 46 (2), 107-126. 